www.nature.com/pj

\title{
Crystallization kinetics of polypropylene containing a sorbitol nucleating agent
}

\author{
Satoshi Katsuno ${ }^{1}$, Masahiro Yoshinaga ${ }^{1}$, Shinichi Kitade ${ }^{2}$, Yusuke Sanada ${ }^{3}$, Isamu Akiba ${ }^{3}$, Kazuo Sakurai ${ }^{3}$ \\ and Hiroyasu Masunaga ${ }^{4}$
}

We carried out an isothermal crystallization experiment on isotactic polypropylene (iPP) containing 0.2 and $3.0 \%$ of 1,3:2,4-bis( $p$-methylbenzylidene)sorbitol (PDTS) as a nucleation additive. Compared with neat iPP, the induction period before crystallization was markedly shortened, and thus the quench depth became shallower by $\sim 15{ }^{\circ} \mathrm{C}$. Avrami analysis showed that the Avrami exponent was very sensitive to temperature; it decreased from 3.5-4 to 2 with a decrease in the crystallization temperature $T_{\mathrm{c}}$ (that is, an increase in the cooling depth) of only $4{ }^{\circ} \mathrm{C}$, in contrast with iPP, where the exponent was 4 for all $T_{\mathrm{c}}$. Small-angle X-ray scattering showed an upturn at low $q$ only for iPP/PDTS, suggesting that the gel network made from PDTS was formed before iPP crystallization. This study shows that the iPP/PDTS crystallization kinetics and its temperature sensitivity were determined by the competition between two events: PDTS network growth and iPP crystallization.

Polymer Journal (2013) 45, 87-93; doi:10.1038/pj.2012.199; published online 14 November 2012

Keywords: crystallization; polypropylene; sorbitol; nucleating agent; synchrotron X-ray; scattering; SAXS

\section{INTRODUCTION}

Controlling crystalline morphology is of essential importance in processing isotactic polypropylene (iPP) because its mechanical and optical properties depend on the size and number of the crystallites. $^{1,2}$ It is well known that nucleating additives have a remarkable role in changing the crystalline morphology. ${ }^{2}$ Among them, sorbitol derivatives (SDs), such as 1,3:2,4-bis( $p$ methylbenzylidene)sorbitol (PDTS; see the chemical structure in Figure 1a), have been used for this purpose for decades. ${ }^{3,4}$ The thermodynamic interpretation of their role is that SDs decrease the crystalline nucleation-free energy in molten iPP and thus decrease the depth of the supercooling temperature necessary for nucleus formation. In terms of crystallization kinetics, it is believed that SDs act as a crystallization nucleus or as an initiator and dramatically increase the population of the precursors for crystallization. According to Kim et al., ${ }^{5}$ who examined the isothermal crystallization kinetics of $\mathrm{iPP} / \mathrm{SD}$ using differential scanning calorimetry, the number of effective nuclei in $\mathrm{iPP} / \mathrm{SD}$ was estimated to be $3 \times 10^{2}-10^{5}$ times larger than that in the neat iPP. Such a large number of nuclei evidently increases the number of spherulites and thus markedly reduces the size of each spherulite. When the spherulite size becomes less than the wavelength of visible light, the finished product becomes transparent. Although the degree of crystallinity is not increased with SDs, the mechanical strength and thermal distortion temperature are improved, due to a structure in which a large number of small crystallites are uniformly dispersed in the finished product. ${ }^{2}$

The molecular mechanism of SD acceleration of iPP crystallization is understood as follows. An SD dissolves in an iPP melt at a high temperature and subsequent cooling causes the SD to precipitate and form a network of fine filaments in the iPP melt. ${ }^{4,6}$ Upon further cooling below the crystallization temperate of iPP, an iPP crystalline lamella grows epitaxially from the surface of the network. Such epitaxial growth is more spatially confined than normal crystalline growth in a molten bulk; thus, its kinetics should exhibit different characteristics from the normal growth. Fillon et al. ${ }^{7}$ carried out differential scanning calorimetry and concluded that SDs contribute to only half of the nucleation relative to self-nucleated iPP. If their conclusion is true for all temperatures, such bimodal crystallization should be reflected in the crystallization kinetics. As far as we know, there are no isothermal crystallization studies for iPP/SD with simultaneous wide-angle X-ray scattering (WAXS) and small-angle X-ray scattering (SAXS), where WAXS and SAXS denote wide and small angle X-ray scattering, repsectively, although there are several studies with differential scanning calorimetry. 3,5

Kristiansen et al. ${ }^{6}$ constructed a phase diagram for mixtures of 1,3:2,4-bis(3,4-dimethylbenzylidene)sorbitol (that is, a family of SDs different from PDTS) and iPP. In the SD concentrations between

\footnotetext{
${ }^{1}$ Products Technical Center Two, Japan Polypropylene Corporation, Yokkaichi, Mie, Japan; ${ }^{2}$ Research and Development Division, Japan Polychem Corporation, Yokkaichi, Mie, Japan; ${ }^{3}$ Department of Chemistry and Biochemistry, University of Kitakyushu, Fukuoka, Japan and ${ }^{4}$ Japan Synchrotron Radiation Research Institute (JASRI/SPring-8), Sayo, Hyogo, Japan

Correspondence: Dr/Professor I Akiba or Professor K Sakurai, Department of Chemistry and Biochemistry, University of Kitakyushu, 1-1 Hibikino, Wakamatsu, Fukuoka 808-0132, Japan. 
a<smiles>Cc1ccc(C2O[C@@H]3COC(c4ccc(C)cc4)O[C@]3(O)[C@](C)(C(O)O)O2)cc1</smiles>

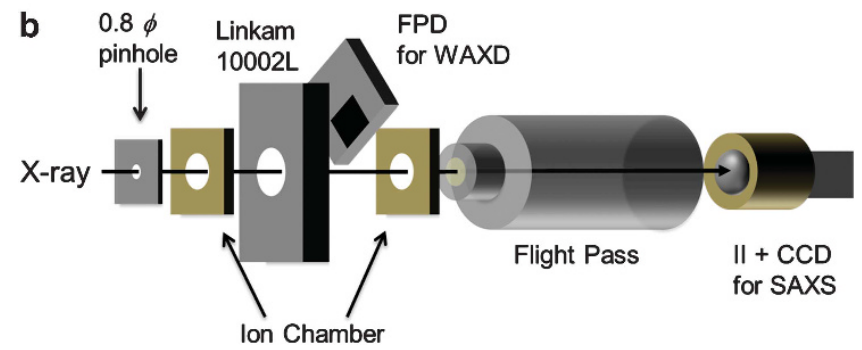

Figure 1 The chemical structure of PDTS (a) and the experimental setup for the WAXS and SAXS measurements at SPring-8 BL03XU (FSBL) (b).

$\sim 0.2$ and $1.0 \mathrm{wt} \%$, iPP and SD formed a homogeneous liquid $\left(\mathrm{L}_{0}\right)$ at high temperatures, and upon cooling, the SD crystallized before the polymer crystallization. During this SD crystallization, its crystallite grew into a micro filament dispersed in the iPP. The filament had a diameter of $<20-50 \mathrm{~nm}$ and was dispersed in the polymer matrix. Kristiansen et al. concluded that because these precipitated filaments provide an extremely large surface area, the iPP crystallization is markedly accelerated. When the SD concentration was $>2.0 \mathrm{wt} \%$, a liquid/liquid macrophase separation $\left(\mathrm{L}_{1}\right.$ and $\left.\mathrm{L}_{2}\right)$ was observed, where $\mathrm{L}_{1}$ and $\mathrm{L}_{2}$ are the SD-poor and SD-rich phases, respectively. Upon cooling, the $\mathrm{SD}$ crystallized in the $\mathrm{L}_{2}$ and in the $\mathrm{L}_{1}$ domains. As the size of the $\mathrm{L}_{2}$ domain was almost the same as the wavelength of visible light, the finished product became turbid.

Kobayashi et al. ${ }^{4,8-10}$ proposed a slightly different model from Kristiansen et al. ${ }^{6}$ Upon cooling from $\mathrm{L}_{0}$, the concentration fluctuation of the SD is enhanced by the spinodal decomposition, and the mixture of $\mathrm{L}_{1}$ and $\mathrm{L}_{2}$ form a bicontinuous structure. When the $\mathrm{SD}$ concentration reaches a certain critical value, the SD forms long needle crystals in the $\mathrm{L}_{2}$ phase, and these crystal filaments form a percolation-type network. Crystallization of the SD freezes the time development of the spinodal decomposition of both phases. Consequently, the morphology of the $\mathrm{L}_{1}$ and $\mathrm{L}_{2}$ domains depends strongly on the cooling conditions. Rapid cooling can keep the $\mathrm{L}_{2}$ domain size small enough to ensure the phase is transparent and allow the domain shape to maintain bi-continuity. Takenaka et al. ${ }^{8,10}$ showed that the SD percolation network forms a self-similar structure with a mass-fractal dimension and that the lower limit of the network was $<20 \mathrm{~nm}$, which was determined from the excluded volume effects of the SD crystal filament.

The appearance of the finished iPP is determined by the SD concentration and the cooling rate. ${ }^{4,6}$ For example, slow cooling with $1 \%$ SD results in a significant reduction in clarity when compared with rapid cooling. As the cooling rate of the inner area is decreased with an increase in thickness, it is still difficult to produce clear thick products by injection molding. The cooling rate dependency and resultant morphological diversity should be related to the competition between three events: iPP crystallization, SD phase separation and SD filament formation. To understand and clarify such a complicated system, it is important to study the crystallization kinetics for the SD/iPP system with simultaneous WAXS and SAXS. The insights and information obtained from these studies should be useful for industrial applications.

The third generation of synchrotron beamlines ${ }^{11}$ made it possible to carry out accurate, simultaneous WAXS and SAXS from polymers because they can provide very bright and stable X-ray beams with extreme accuracy. The aim of this study is to investigate the isothermal crystallization kinetics of $\mathrm{PP} / \mathrm{SD}$ by using a synchrotron light source to explore the kinetics in terms of structural development.

\section{EXPERIMENTAL PROCEDURE}

\section{Materials and molecular weight characterization}

We used commercial grade homo linear iPP polymerized with a Zieglar-Natta catalyst obtained from the Japan Polypropylene Corporation (Tokyo, Japan). The weight-averaged molecular weight $\left(M_{\mathrm{W}}\right)$ of the iPP sample, determined by size exclusion chromatography, was $2.8 \times 10^{5}$, and the molecular weight distribution, defined by $M_{\mathrm{W}} / M_{\mathrm{N}}$, was 4.6 , where $M_{\mathrm{N}}$ is the number-averaged molecular weight. PDTS was purchased from New Japan Chemical Co., Ltd. (Osaka, Japan). We mixed the iPP sample and the PDTS (0.2 and $3.0 \mathrm{wt} \%$ of iPP), compounded them in a twin-screw extruder (Technovel Corporation KZW15 mm, Osaka, Japan) at $230^{\circ} \mathrm{C}$ and molded them into a plate with a thickness of $\sim 1 \mathrm{~mm}$ at $230^{\circ} \mathrm{C}$ for $2 \mathrm{~min}$. These two samples were denoted iPP/ PDTS0.2 and iPP/PDTS3.0. iPP/PDTS0.2 was transparent whereas iPP/ PDTS3.0 was rather turbid due to the macrophase separation of the PDTS. By changing the cooling and heating rates during the differential scanning calorimetry, the equilibrium melting temperature $\left(T_{\mathrm{m}}{ }^{0}\right)$ was determined to be $196.5^{\circ} \mathrm{C}$ for the three samples, which include the neat iPP.

\section{Time-resolved X-ray measurements}

Time-resolved SAXS and WAXS measurements were carried out at the beam line BL03XU in the synchrotron radiation facility at SPring-8, Hyogo, Japan. ${ }^{11}$ The experimental setup is schematically shown in Figure $1 \mathrm{~b}$. The sample-to-detector distance was $1.5 \mathrm{~m}$ for the SAXS and $0.07 \mathrm{~m}$ for the WAXS measurements. SAXS and WAXS intensities were measured with a cooled CCD (Hamamatsu Photonics V7739P + ORCA R2, Hamamatsu, Shizouka, Japan) and a flat panel detector (Hamamatsu Photonics C9728DK-10). The flat panel detector was installed on the same stage and tilted to $45^{\circ}$. The X-ray scattering and diffraction data were acquired every $3 \mathrm{~s}$. The background and dark data were subtracted from the raw data. The invariant $Q$ was determined from the SAXS profile by numerically integrating the intensity for all of the measured angles, and the relative degree of crystallinity $X_{\mathrm{c}}$ was calculated from the peak area of the $\alpha$ form (110) diffraction peak after the amorphous background was subtracted.

\section{Temperature protocol}

The sample was loaded into a Linkam heating/cooling cell (Linkam, Surrey, UK) and heated to $250^{\circ} \mathrm{C}$ to melt the sample. After $3 \mathrm{~min}$, the sample was cooled to a given crystallization temperature with a cooling rate of $-60{ }^{\circ} \mathrm{C} \mathrm{min}^{-1}$. It took $\sim 120 \mathrm{~s}$ to reach the crystallization temperature.

\section{Theoretical treatment of isothermal crystallization}

According to the method from Avrami ${ }^{12,13}$ on treating the simple case of perfect crystalline bodies growing from randomly formed nuclei in a supercooled melt, the degree of crystallinity $X_{\mathrm{c}}(t)$ at time $t$ is generally given by:

$$
X_{\mathrm{c}}(t)=\int_{0}^{t} N(\tau)\left[1-X_{\mathrm{c}}(\tau)\right] v(\alpha, \tau) \mathrm{d} \tau .
$$

Here, $N(\tau)$ is the nucleation rate for the crystalline formation, and $v(\alpha, \tau)$ is the crystallite volume at time $\tau$ for a given crystallite whose nucleus formed at time $\alpha$. This model does not account for collisions between crystallization growth fronts, and thus only holds for the initial stages of crystallization. For simplicity, we consider two nucleation mechanisms: instantaneous and homogeneous nucleation. In the former case, all nuclei form at $\alpha=0$. In the latter case, nuclei form at a constant rate. For small degrees of crystallinity, Equation (1) is then simplified to

$$
-\ln \left[1-X_{\mathrm{c}}(t)\right]^{n}
$$



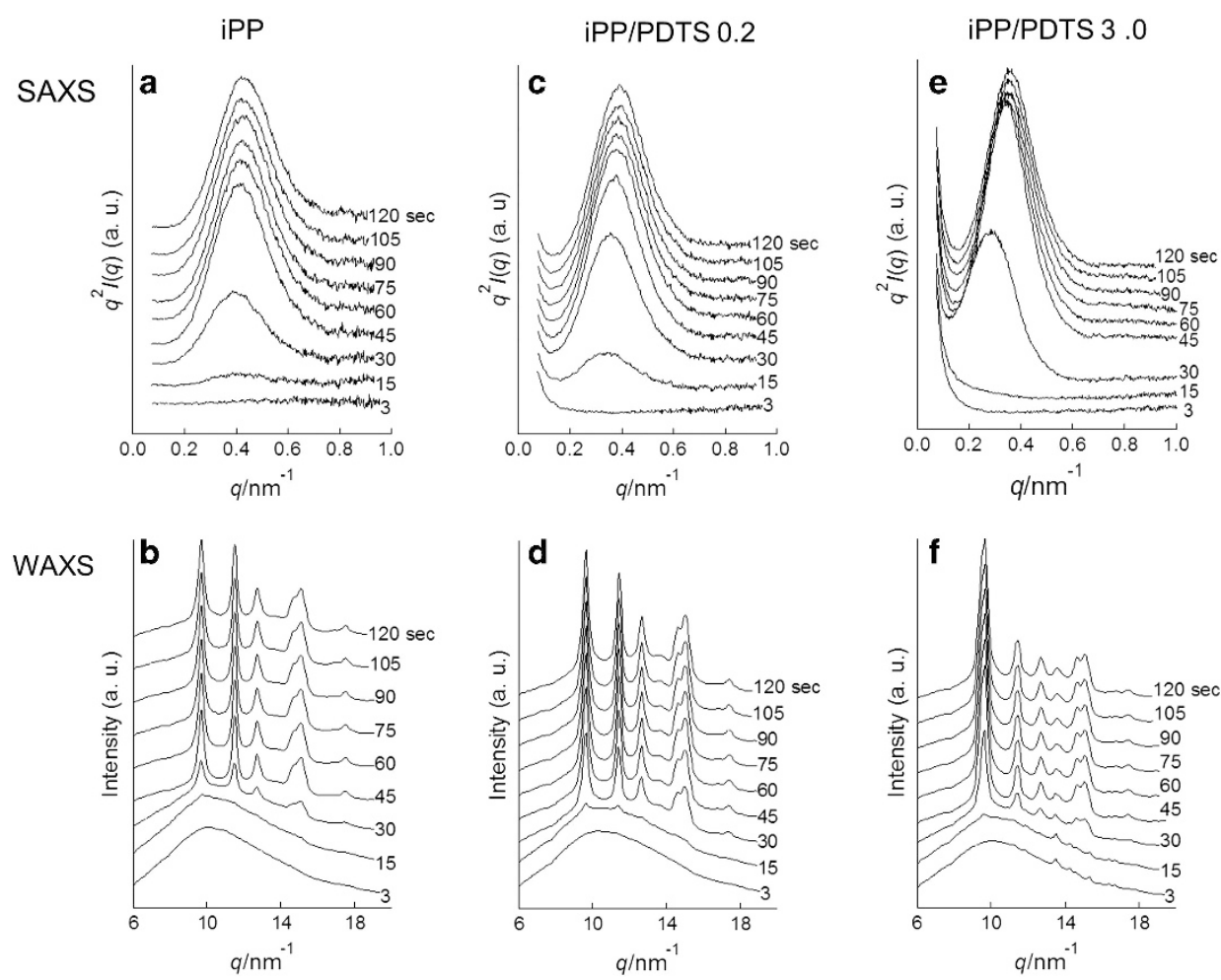

Figure 2 The time development of the WAXS and SAXS profiles collected during the isothermal crystallization of the neat iPP at $137^{\circ} \mathrm{C}$ and of the iPP/ PDTS at $151{ }^{\circ} \mathrm{C}$. For convenience in comparing the profiles, we chose data with similar induction periods. (a) SAXS for iPP without PDTS, (b) WAXS for iPP without PDTS, (c) SAXS for iPP containing 0.2 wt\% PDTS, (d) WAXS for iPP containing 0.2 wt\% PDTS, (e) SAXS for iPP containing 3.0 wt\% PDTS, and (f) WAXS for iPP containing 3.0 wt $\%$ PDTS.

Here, $n$ is the Avrami exponent, which is related to the nucleation mechanism and to the geometry of the crystal growth.

For Avrami analysis, we used the degree of crystallinity determined from WAXS. It should be noted that $t$ in Equations $(1$ and 2$)$ is the time elapsed since crystallization had started, and thus $t=t_{\mathrm{c}}-t_{\mathrm{i}}$, where $t_{\mathrm{c}}$ is the elapsed time since the sample temperature reached $T_{\mathrm{c}}$, and $t_{\mathrm{i}}$ is the induction period. The value of $t_{\mathrm{i}}$ was determined by extrapolating $X_{\mathrm{c}}$ to 0 using the initial increase in $X_{\mathrm{c}}$. There was some experimental ambiguity in determining $t_{\mathrm{i}}$, as mentioned by Wang et al. ${ }^{14}$ We adjusted the extrapolation to obtain a linear relation in the initial stages when we constructed the $-\ln \left[1-X_{c}(t)\right]$ vs $t$ plot.

\section{RESULTS AND DISCUSSION}

\section{Isothermal crystallization}

Typical time-resolved SAXS and WAXS profiles taken during the isothermal crystallization of iPP are shown in Figures $2 a$ and $b$, where $T_{\mathrm{c}}=137^{\circ} \mathrm{C}$. The neat iPP showed the formation of the typical $\alpha$ crystalline form in the WAXS data and showed the growth of the long period of the stacked iPP crystal lamellae in the SAXS data. Under isothermal crystallization at $137^{\circ} \mathrm{C}$, crystallization was clearly detected by SAXS at $15 \mathrm{~s}$, and there was a small delay in the detection by WAXS. This delay is interpreted as the early SAXS peak, in our case before $15 \mathrm{~s}$, being correlated with the density fluctuations of the polymeric melts before crystallization. ${ }^{14,15}$ Figure 3 shows the time dependencies of the normalized invariant $(Q)$ and of the normalized degree of crystallinity $\left(X_{c}\right)$ for iPP. $Q$ and $X_{c}$ increased with time in essentially the same manner. Figure 4a shows the Avrami plot with an Avrami exponent of $n=4$. This means that the crystallization of iPP in this temperature range was governed by a combination of continuous nucleation and three-dimensional-free growth, as reported previously. ${ }^{16}$

Figures $2 \mathrm{c}$ and $\mathrm{d}$ show the SAXS and WAXS profiles for iPP/ PDTS0.2 at $T_{\mathrm{c}}=151{ }^{\circ} \mathrm{C}$. We chose this $T_{\mathrm{c}}$ to be different from that of the iPP sample so that the crystallization induction periods for both samples are almost equal. One may think that we should compare the crystallizations at the same cooling depth. However, as discussed in more detail later, the crystallization rate and the induction period were too different to be drawn on the same plot. The SAXS data showed that there was an upturn at low $q$, while this result was not observed for iPP. The presence of such an upturn could be related to the network structure of PDTS (discussed later). At $15 \mathrm{~s}$, the SAXS data showed the formation of the long period of iPP, and the WAXS data showed the crystalline diffraction peaks. Comparing the iPP/ PDTS0.2 with the neat iPP, it can be confirmed that crystallization of iPP/PDTS0.2 was markedly accelerated as a result of the presence of PDTS. There was a new peak in iPP/PDTS0.2 data at $q=13.5 \mathrm{~nm}^{-1}$, which can be assigned to $\gamma$ phase crystals or to the crystalline diffraction of PDTS (see WAXS at $t=3 \mathrm{~s}$ for iPP/PDTS3.0). ${ }^{17}$

Figures $2 \mathrm{e}$ and $\mathrm{f}$ show the results from the iPP/PDTS3.0 sample at $T_{\mathrm{c}}=151^{\circ} \mathrm{C}$. Macrophase separation was observed in the molten iPP/ PDTS3.0, but it seemed that the crystallization kinetics were not affected by this. When we examined the PDTS concentration dependence of the crystallization rate and other behaviors in the PDTS concentration range of $<3 \mathrm{wt} \%$, the crystallization rate continuously increased with increasing amounts of PDTS, and we did not observe any discontinuity in the rate or in other behaviors. We presumed that if macrophase separation affects crystallization, we should be able to see certain discontinuities when we passed the boundary between the homogeneous and the bimodal phase. We consider the crystallization to have been mainly determined by the PDTS dispersed in the PDTS poor domain $\left(\mathrm{L}_{1}\right)$; thus, the kinetics of crystalline growth in iPP/PDTS3.0 can be discussed in the same manner regardless of the presence of macrophase separation. WAXS 


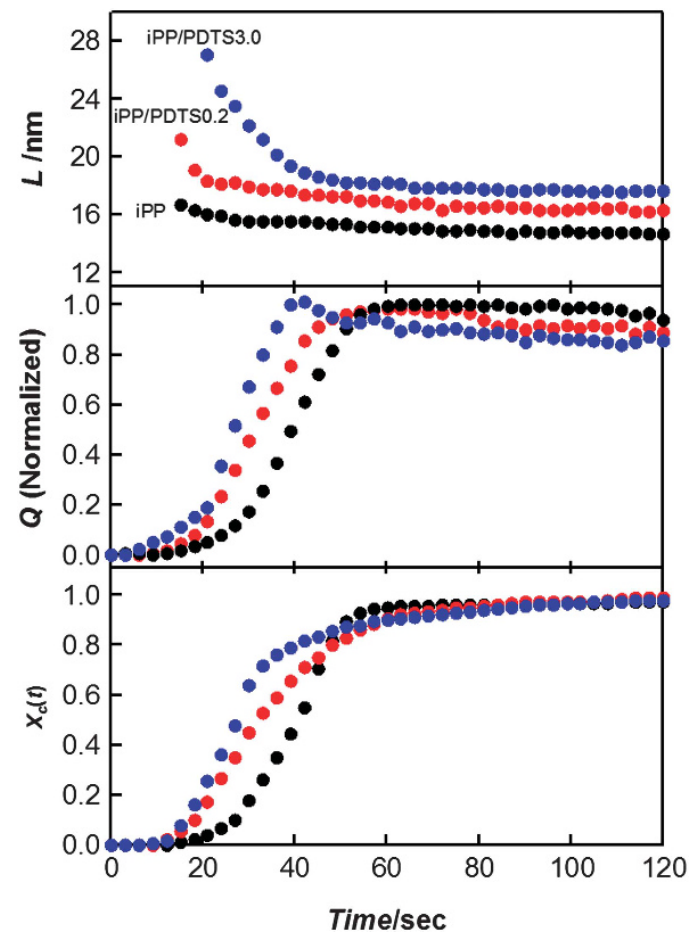

Figure 3 The time development of the long period $(L)$ determined from the peak top position from SAXS, the normalized invariant $(Q)$ determined from SAXS and the degree of crystallinity $\left(X_{c}\right)$ determined from WAXS. The time $t_{c}$ is the elapsed time since the cell temperature reached the crystallization temperature.

at $t=3 \mathrm{~s}$ showed that there was some amount of crystalline PDTS present at $250^{\circ} \mathrm{C}$, and upon cooling, their peak intensities did not increase significantly. At $q=13.5 \mathrm{~nm}^{-1}$, there was a clearer peak observed for the iPP/PDTS3.0 sample than for the iPP/PDTS0.2 sample. Again, this peak can be assigned to $\gamma$ crystals; the crystalline diffraction of PDTS was indistinguishable from $\gamma$ crystals of iPP.

Comparison of the time development of the crystallization Figure 2 compares the time development of the long period $(L)$ determined from the peak top position from SAXS, the normalized invariant $(Q)$ determined from SAXS and the degree of crystallinity $\left(X_{\mathrm{c}}\right)$ determined from WAXS for the three samples. $Q$ and $X_{\mathrm{c}}$ increased in a similar manner in the initial stages, indicating that both parameters are reflecting crystalline growth. In the later stages, the iPP/PDTS samples showed that $Q$ reached a plateau earlier than $X_{\mathrm{c}}$ and slightly decreased after $60 \mathrm{~s}$, especially with the iPP/PDTS3.0 sample, which showed a maximum at $40 \mathrm{~s}$. We did not see such a decrease in $Q$ for iPP. Currently, we do not have an explanation for such a discrepancy occurring only in the iPP/PDTS samples. This discrepancy indicates that $Q$ is not determined only by the iPP crystals; thus, we assume that the PDTS network could contribute to the decrease in the SAXS intensity in the later stages. For the neat iPP, $L$ hardly changed, although the SAXS peak intensity markedly increased (see Figure 2a). The same result was reported previously. ${ }^{14,15}$ According to these authors, this phenomenon is explained as follows. $L$ is almost equal to the average distance between the two phases with different densities, which are generated by spinodal decomposition before crystallization. Crystallization precursors are created in the higher density domain, and once crystallization starts there, the time development of the
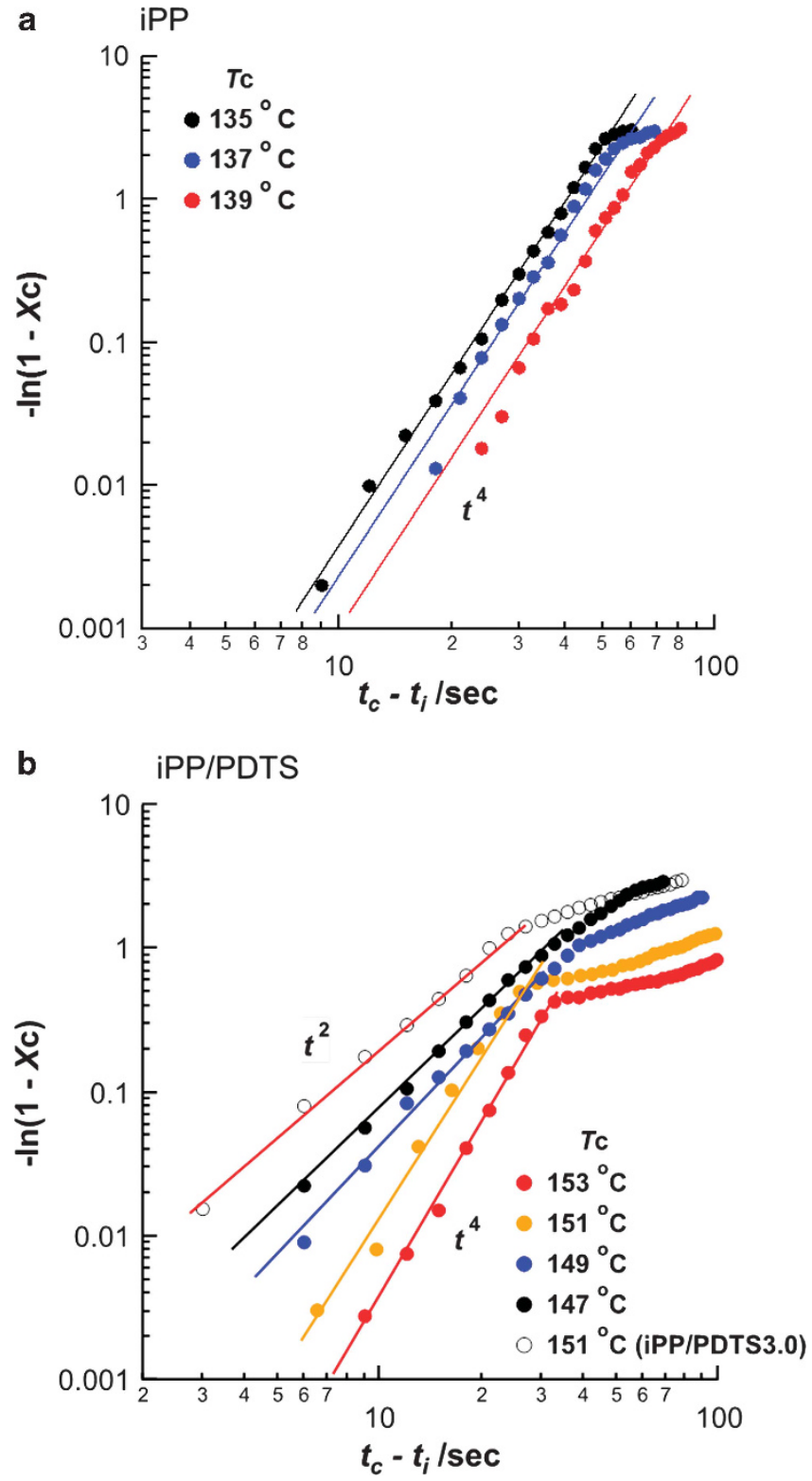

Figure 4 Comparison of the Avrami plots for the three samples. The filled circles in the lower panel are for the iPP/PDTSO.2 and the unfilled one is for the iPP/PDTS3.0. (a) iPP without PDTS and (b) iPP with PDTS.

spinodal decomposition is frozen; thus, the value of $L$ hardly changed. Contrary to neat iPP, there was a relatively large decrease observed in $L$ for iPP/PDTS0.2 and iPP/PDTS3.0. In addition, the value of $L$ in the later stages was longer for iPP/PDTS than for iPP. These two facts, decreasing $Q$ and increasing $L$ in the iPP/PDTS samples, suggest that a different mechanism governs $L$.

\section{Induction period}

The 'induction period' is defined as the period before the observation of crystallization. According to classical nucleation and growth theories, the induction period is the time required for the formation of a nucleus or its precursors. Recently, several authors have shown experimentally that the density fluctuation driven by spinodal decomposition creates the precursors of crystallization. ${ }^{14,15,18,19}$ There is still no clear molecular picture for the crystallization 


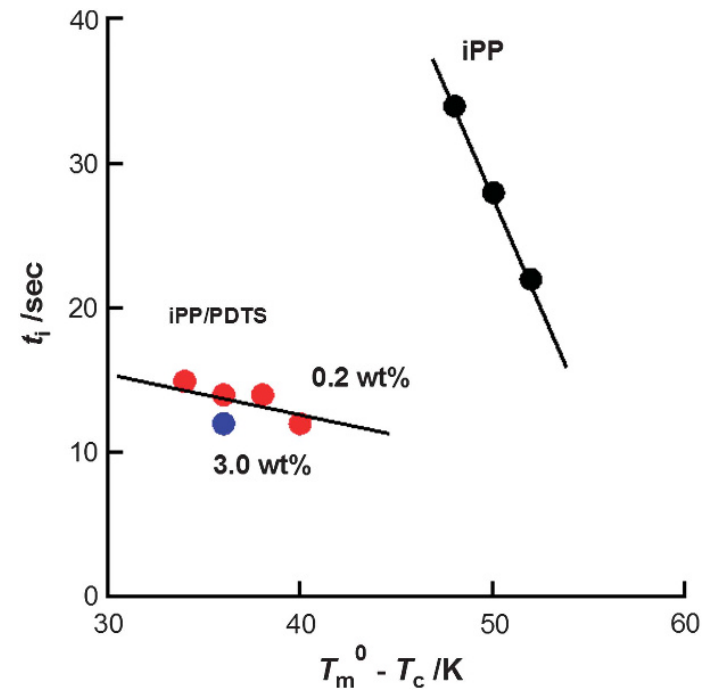

Figure 5 Quench depth dependence of the induction period $\left(t_{\mathrm{i}}\right)$.

precursors or nuclei. Reddy et al. ${ }^{15,16}$ carried out time-resolved FTIR, as well as WAXS, during the isothermal crystallization of iPP and proposed that the crystallization precursors for iPP could be an aggregation of the short iPP helices. These helices are dynamically moving, and their stacking is not strong enough to exhibit a WAXS signal, but they can be detected with FTIR. To form the $\alpha$ phase crystal or its precursors, the characteristic iPP $3_{1}$ helices have to be arranged in an antiparallel manner. Therefore, the aggregate of short iPP helices eventually has to adopt such an antiparallel structure to grow iPP crystals. The rather long induction period of iPP may be understood in this framework because it could take a certain amount of time to reach a critical concentration and size to yield such crystallization precursors as well as for the aggregate helices to rearrange their alignment.

Figure 5 compares the temperature dependence of the induction period $\left(t_{\mathrm{i}}\right)$ between iPP/PDTS and iPP. As expected, the induction period for iPP markedly decreased with an increase in the depth. This is because the instability of molten iPP becomes more significant at lower temperatures; thus, the density fluctuation needed to create the precursors increases. Note that $t_{\mathrm{i}}$ was considerably shorter for iPP/ PDTS than for iPP and that the cooling depth was very shallow. Another interesting point about iPP/PDTS is that $t_{\mathrm{i}}$ decreased with an increase in the depth, but its temperature dependency was much weaker than in iPP. These facts indicate that the presence of PDTS mainly determined the precursor formation instead of the density fluctuation. Although there is a large concentration difference between iPP/PDTS0.2 and iPP/PDTS3.0, as well as the presence of macrophase separation in iPP/PDTS3.0, the difference in Figure 5 was small. This means that even a small amount of PDTS can markedly reduce the induction period. In fact, as many reports mentioned, $3,4,20$ the maximum effect of the SD additives occur at concentrations $\sim 0.3 \%$, and further addition of SD does not show much improvement.

\section{Avrami analysis}

Figure 4 compares the Avrami plots for the three samples presented in Figure 2. iPP showed a slope of 4 , again indicating continuous nucleation and three-dimensional growth. There was not much difference between the two iPP/PDTS samples, but they showed quite a different behavior from iPP; the slope was approximately two in the
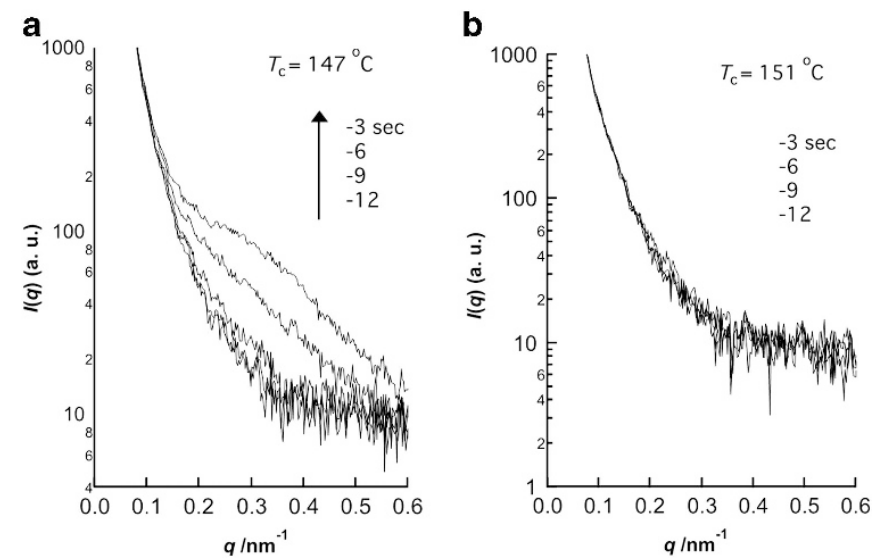

Figure 6 The time development of the SAXS data at low $q$ before crystallization. The elapsed time indicated is calculated from when the crystallization starts. The crystallization temperatures were $147^{\circ} \mathrm{C}$ (a) and $151^{\circ} \mathrm{C}(\mathbf{b})$.

early stages and further decreased to $<1$ in the later stages. The lower exponent in the early stages is ascribed to a combination of instantaneous nucleation and specially confined crystalline growth, and thus can be related to epitaxial-type crystalline growth from PDTS fibers. The lower crystallization rate in the later stages may be interpreted as follows. The number of crystalline precursors is much larger in iPP/PDTS than in iPP, and thus collisions between the crystalline growth fronts should occur more frequently in iPP/PDTS than that in iPP. These collisions reduce the crystallization rate.

Figure 4 also shows the quench depth dependence of the Avrami plots for iPP/PDTS0.2. With an increase in $T_{\mathrm{c}}$ (that is, a decrease in the quench depth), the slope increased from $n=2$ to $n=3.5-4$ at $T_{\mathrm{c}}=153^{\circ} \mathrm{C}$, showing that a substantial change is occurring in the kinetics over a small temperature range. Note that at $T_{\mathrm{c}}=153^{\circ} \mathrm{C}$ the induction period was considerably shorter when compared with iPP, but $n$ was close to 4 , indicating three-dimensional growth. These features can be interpreted as the large amount of precursors existing before crystallization reducing the induction period, but the crystalline growth from these precursors was not spatially confined at $153^{\circ} \mathrm{C}$. On the other hand, the spatial confinement dominated the crystallization at $147^{\circ} \mathrm{C}$. One of the possible explanations for such differences in behavior is that the subtle temperature difference between 147 and $153^{\circ} \mathrm{C}$ determines what mechanism dominates the competition between the iPP crystallization and the network formation of PDTS.

\section{Upturn at low $q$ in the SAXS data and its interpretation}

As mentioned in Figure 2, there was an upturn observed at low $q$, and this can be attributed to the density fluctuation of PDTS because we did not see such an upturn for iPP. Figure 6 compares the time development of the SAXS data upturn between $T_{\mathrm{c}}=147$ and $151^{\circ} \mathrm{C}$ for iPP/PDTS0.2. There was essentially no change at $151^{\circ} \mathrm{C}$, but an appreciable time development was observed at $147^{\circ} \mathrm{C}$. Reddy et al..$^{15}$ observed a similar change in the SAXS data for iPP itself when molten iPP was cooled to $126^{\circ} \mathrm{C}$ (their equilibrium melting temperature was $196.5^{\circ} \mathrm{C}$ ). They interpreted their change as a bimodal type density fluctuation and used the Debye-Bueche relation to obtain a correlation length, which describes the characteristic length between neighboring domains. Although the apparent change in Figure 6a 

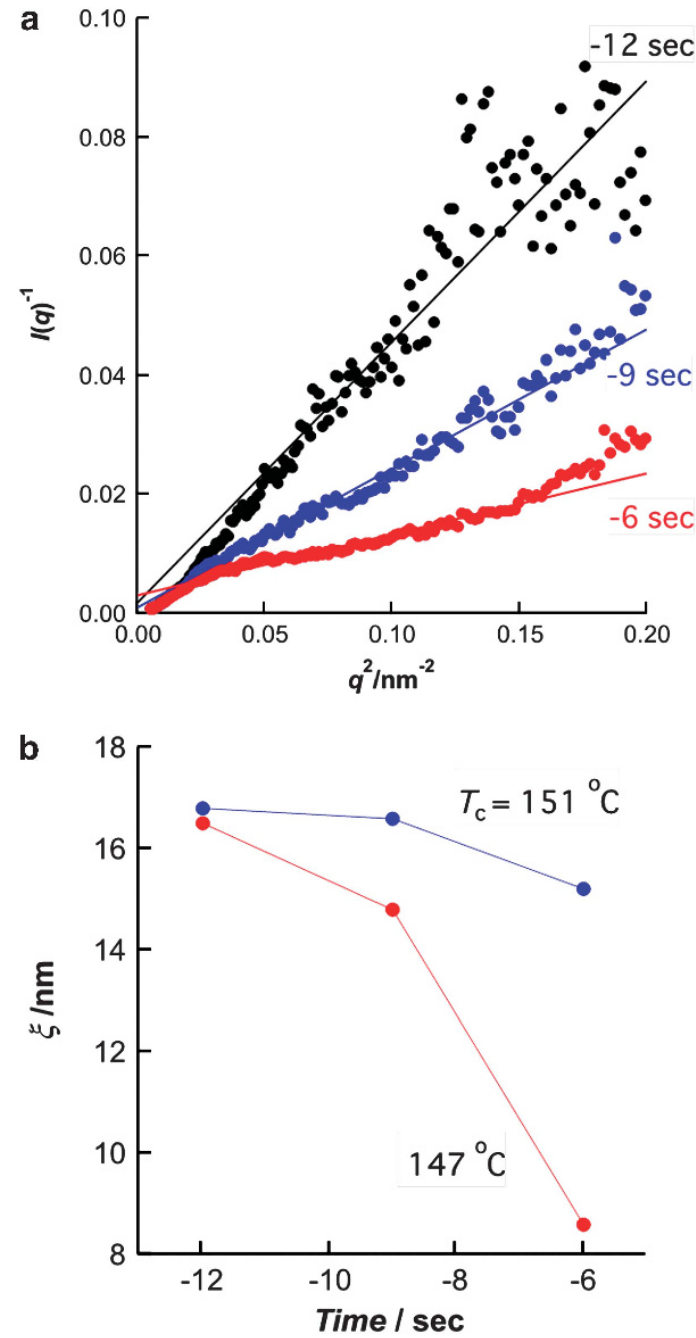

Figure 7 The time development of the $/(q)^{-1}$ vs $q^{2}$ profile at $T_{\mathrm{c}}=147^{\circ} \mathrm{C}$ (a) and comparison of the time development of the Ornstein-Zernike correlation length $\xi$ at $T_{\mathrm{c}}=147$ and $151{ }^{\circ} \mathrm{C}$ (b) for the iPP/PDTSO.2 before crystallization.

was very similar to that of Reddy et al., we presume that our observation is not related to the phase separation of molten iPP. This is because there is no such phenomenon observed in iPP in our system and because the upturn in the SAXS data was enhanced by increasing the PDTS concentration.

Takenaka et al. ${ }^{8,10}$ investigated the time evolution of the dynamic viscosity for a PDTS mixture $(2.5 \mathrm{wt} \%)$ in a polystyrene melt at $185^{\circ} \mathrm{C}$. When the mixture reached the gelation point, the storage and loss shear moduli $\left(G^{\prime}\right.$ and $\left.G^{\prime \prime}\right)$ were described by the power law $G^{\prime} \sim G^{\prime \prime} \sim \omega^{2 / 3}$, showing that the gel network can be described by the percolation model. They also showed that this power law (that is, the network structure) was independent of the cooling depth. Kobayashi and Hashimoto showed that PDTS forms almost the same network regardless of the matrix material, including low-molecular organic solvents. Therefore, it is reasonable to assume that a percolation-type gel network, as described by Takenaka et al., ${ }^{8,10}$ was formed during cooling from the molten iPP. Although the final morphology of the gel networks is represented by a percolation network with a massfractal dimension, it is unlikely that such percolation appears at the early beginnings of the aggregation or of the crystallization of PDTS.

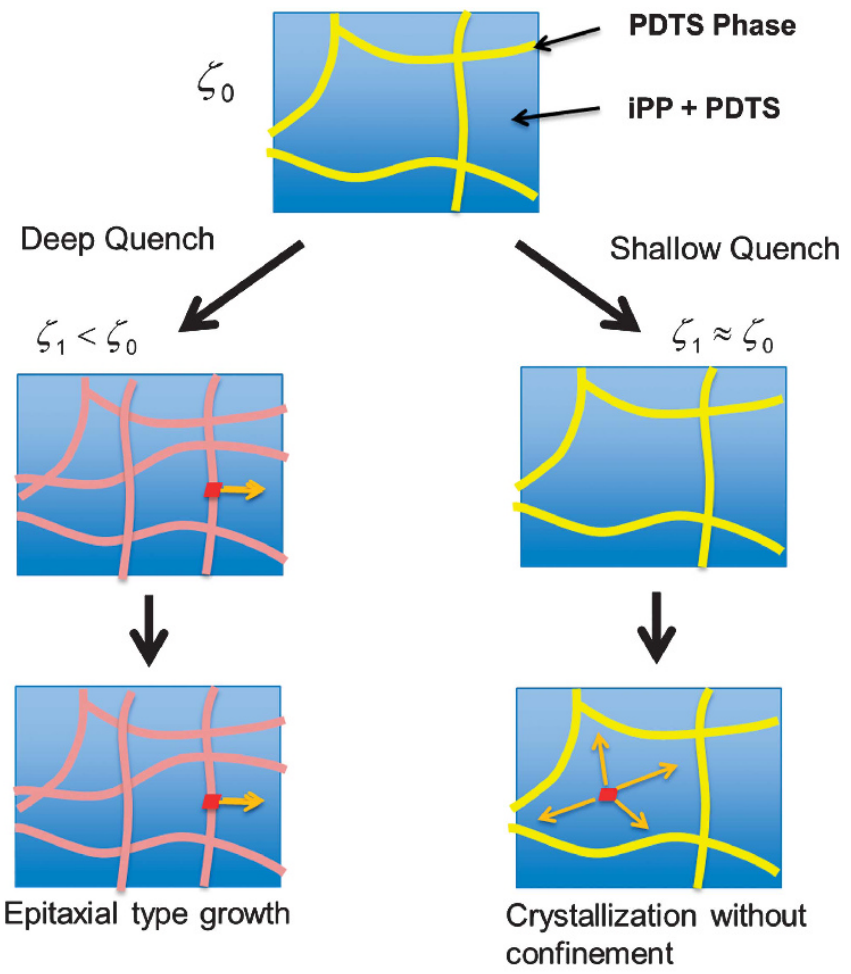

Fresh surface

Figure $8 \mathrm{~A}$ schematic model to explain the crystallization kinetics at different temperatures for iPP/PDTS.

The initial structure of PDTS network may be described by the large fluctuation of the PDTS concentration or by a PDTS gel network with a characteristic length. To clarify the initial structure, we need to measure the SAXS at a lower $q$ range; however, this may be a subject for the future.

The aforementioned structures can be described in terms of an exponentially decaying correlation function $\exp (-r / \varsigma),{ }^{21}$ where $r$ and $\zeta$ are the average distance between structures in real space and the correlation length to determine how long structural correlations are maintained. Therefore, we analyzed the scattering data upturns in Figure 6 with the Ornstein-Zerniike equation, which can be directly derived from the aforementioned correlation function:

$$
I(q) \propto \frac{I(0)}{1+q^{2} \zeta^{2}} .
$$

Figure 7a plots $I(q)^{-1}$ against $q^{2}$. The value of $\zeta$ can be determined from the slope of each plot, and the results are plotted against time in Figure 8 b. At $151^{\circ} \mathrm{C}$, $\zeta$ hardly changed, whereas it decreased with increasing time at $147^{\circ} \mathrm{C}$. This result can be interpreted as the PDTS network not developing well or rapidly at a higher temperature, whereas it can grow rapidly at a lower temperature.

\section{Relation between the PDTS network and the iPP crystallization dynamics}

Combining the results mentioned above, we can propose a model to explain the kinetics of iPP/PDTS as follows. At a shallow quench, the PDTS network formation is relatively slow and thus iPP crystallization starts before the network formation is completed. There is a large amount of PDTS dispersing in the molten iPP, and these PDTS molecules, or their aggregate, can act as the nuclei of crystallization 
even though they do not form filaments or gel networks. At a deep quench, the network formation of the PDTS filaments is relatively fast and the freshly formed surface of the filaments can be a good nucleus for crystallization. Once the iPP crystal grows from the filament surface, it suffers spatial confinement, and thus the Avrami exponent decreases. These models are schematically illustrated in Figure 8.

Kobayashi and Hashimoto proposed a model in which iPP and PDTS are homogenously mixed $\left(\mathrm{L}_{0}\right)$ at a certain high temperature and subsequent cooling induces a phase separation $\left(\mathrm{L}_{1}\right.$ and $\left.\mathrm{L}_{2}\right)$, and PDTS forms a network consisting of PDTS filaments. When we heated iPP/PDTS0.2 and iPP/PDTS3.0 to 280 or $300^{\circ} \mathrm{C}$, we observed the disappearance of the PDTS crystalline peaks in WAXS as well as the disappearance of the upturn in SAXS. After heating these two samples up, we carried out isothermal crystallization at $151^{\circ} \mathrm{C}$. In this case, the crystallization was very slow and we did not see any nucleation effect from the PDTS. This result suggests that to manifest the nucleation effect of PDTS, the PDTS molecules have to form an aggregation. This aggregate may not necessarily be a crystalline form of PDTS because we did not observe its crystalline peak in Figure 2d before the iPP crystallization started. More detailed studies are needed to clarify what is essential in manifesting the nucleation effect of PDTS.

\section{CONCLUSIONS}

We carried out isothermal crystallization experiments for iPP/PDTS after we melted the samples at $250^{\circ} \mathrm{C}$ and cooled them to $T_{\mathrm{C}}=147-$ $151{ }^{\circ} \mathrm{C}$. Compared with neat iPP, the induction period was shorter by $<10 \%$, and the quench depth to start crystallization became shallower by $\sim 15^{\circ} \mathrm{C}$. Avrami analysis showed that there were two regions; in the early stages the Avrami exponent decreased from 3.5-4 to 2 with a decrease in $T_{\mathrm{c}}$ (that is, an increase in the cooling depth) from 151 to $147^{\circ} \mathrm{C}$ of only $4{ }^{\circ} \mathrm{C}$, and in the later stages the exponent became $<1$. This was contrasted with iPP, where the exponent was 4 for all temperatures in the early stages and where the later stages were very short. SAXS showed an upturn in the low $q$ range only for the iPP/PDTS, suggesting that a gel network was made from the PDTS. The upturn increased with time at $147^{\circ} \mathrm{C}$, whereas it hardly changed at $151^{\circ} \mathrm{C}$. These facts indicate that the iPP crystallization kinetics was determined by the balance of two events: PDTS network growth and iPP crystallization.

\section{ACKNOWLEDGEMENTS}

All of the synchrotron X-ray measurements were carried out at the BL-03XU beamline (FSBL) at SPring-8 with the approval of the Japan Synchrotron Radiation Research Institute (2012A7221, 2011B7271, 2011A7221, 2010B7275). We appreciate Miss Naoko Fukuoka for kind support.
1 Moore, E. P. J. Polypropylene Handbook: Polymerization, Characterization, Properties, Processing, Applications (Hanser Publishers, Munich, 1996).

2 Pasquini, N. Polypropylene Handbook. 2nd edn (Hanser Publishers, Munich, 2005).

3 Marco, C., Ellis, G., Gómez, M. A. \& Arribas, J. M. Comparative study of the nucleation activity of third-generation sorbitol-based nucleating agents for isotactic polypropylene. J. Appl. Polym. Sci. 84, 2440-2450 (2002).

4 Kobayashi, T. \& Hashimoto, T. Development of self-assembling nucleators for highly transparent semi-crystalline polypropylene. Bull. Chem. Soc. Jpn 78, 218-235 (2005).

$5 \mathrm{Kim}$, C. Y., Kim, Y. C. \& Kim, S. C. Temperature dependence of the nucleation effect of sorbitol derivatives on polypropylene crystallization. Polym. Eng. Sci. 33, 1445-1451 (1993).

6 Kristiansen, M., Werner, M., Tervoort, T., Smith, P. \& Blomenhofer, M. The binary system isotactic polypropylene/bis(3,4-dimethylbenzylidene)sorbitol: phase behavior, nucleation, and optical properties. Macromolecules 36, 5150-5156 (2003).

7 Fillon, B., Lotz, B., Thierry, A. \& Wittmann, J. C. Self-nucleation and enhanced nucleation of polymers. Definition of a convenient calorimetric 'efficiency scale' and evaluation of nucleating additives in isotactic polypropylene ( $\alpha$ phase). J. Polym. Sci. B: Polym. Phys. 31, 1395-1405 (1993).

8 Takenaka, M., Kobayashi, T., Saijo, K., Tanaka, H., Iwase, N., Hashimoto, T. \& Takahashi, M. Comparison in fractal dimension between those obtained from structure factor and viscoelasticity of gel networks of 1, 3: 2, 4-bis-0-(p-methylbenzylidene)-Dsorbitol in polystyrene melt at gel point. J. Chem. Phys. 121, 3323-3328 (2004).

9 Kobayashi, T., Takenaka, M., Saijo, K. \& Hashimoto, T. Self-assembly and morphology of gel networks in 1, 3: 2, 4-Bis-0-(p-methylbenzylidene)-D-sorbitol/n-dibutylphthalate. J. Colloid Interface Sci. 262, 456-465 (2003).

10 Takenaka, M., Kobayashi, T., Hashimoto, T. \& Takahashi, M. Time evolution of dynamic shear moduli in a physical gelation process of 1, 3: 2, 4-Bis-0-(p-methylbenzylidene)D-sorbitol in polystyrene melt: critical exponent and gel strength. Phys. Rev. E 65, 041401 (2002).

11 Masunaga, H., Ogawa, H., Takano, T., Sasaki, S., Goto, S., Tanaka, T., Seike, T., Takahashi, S., Takeshita, K., Nariyama, N., Ohashi, H., Ohata, T., Furukawa, Y., Matsushita, T., Ishizawa, Y., Yagi, N., Takata, M., Kitamura, H., Sakurai, K., Tashiro, K., Takahara, A., Amamiya, Y., Horie, K., Takenaka, M., Kanaya, T., Jinnai, H., Okuda, H., Akiba, I., Takahashi, I., Yamamoto, K., Hikosaka, M., Sakurai, S., Shinohara, Y., Okada, A. \& Sugihara, Y. Multipurpose soft-material SAXS/WAXS/GISAXS beamline at SPring-8. Polym. J. 43, 471-477 (2011).

12 Avrami, M. Kinetics of phase change. I general theory. J. Chem. Phys. 7, 1103-1112 (1939).

13 Avrami, M. Kinetics of phase change. II transformation-time relations for random distribution of nuclei. J. Chem. Phys. 8, 212-224 (1940).

14 Wang, Z.-G., Hsiao, B. S., Sirota, E. B., Agarwal, P. \& Srinivas, S. Probing the early stages of melt crystallization in polypropylene by simultaneous small- and wide-angle X-ray scattering and laser light scattering. Macromolecules 33, 978-989 (2000).

15 Reddy, K. R., Tashiro, K., Sakurai, T., Yamaguchi, N., Sasaki, S., Masunaga, H. \& Takata, M. Isothermal crystallization behavior of isotactic polypropylene H/D blends as viewed from time-resolved FTIR and synchrotron SAXS/WAXD measurements. Macromolecules 42, 4191-4199 (2009).

16 Reddy, K. R., Tashiro, K., Sakurai, T. \& Yamaguchi, N. Isotope effect on the isotherma crystallization behavior of isotactic polypropylene blends between the deuterated and hydrogenated species. Macromolecules 42, 1672-1678 (2009).

17 Balzano, L., Rastogi, S. \& Peters, G. W. M. Flow Induced crystallization in isotactic polypropylene-1,3:2,4-Bis(3,4-dimethylbenzylidene)sorbitol Blends: Implications on morphology of shear and phase separation. Macromolecules 41, 399-408 (2007).

18 Imai, M., Kaji, K. \& Kanaya, T. Structural formation of poly(ethylene terephthalate) during the induction period of crystallization. 3. Evolution of density fluctuations to lamellar crystal. Macromolecules 27, 7103-7108 (1994).

19 Terrill, N. J., Fairclough, P. A., Towns-Andrews, E., Komanschek, B. U., Young, R. J. \& Ryan, A. J. Density fluctuations: the nucleation event in isotactic polypropylene crystallization. Polymer 39, 2381-2385 (1998).

20 Marco, C., Ellis, G., Gómez, M. A. \& Arribas, J. M. Analysis of the isothermal crystallization of isotactic polypropylene nucleated with sorbitol derivatives. J. Appl. Polym. Sci. 88, 2261-2274 (2003).

21 Ornstein, L. S. \& Zernike, F. Accidental deviations of density and opalescence at the critical point of a single substance. Proc. Akad. Sci. 17, 793 (1914). 